\title{
Feeding the melting pot: inclusive strategies for the multi-ethnic city
}

\author{
Anke Brons $^{1,2} \cdot$ Peter Oosterveer ${ }^{2} \cdot$ Sigrid Wertheim-Heck $^{1,2}$
}

Accepted: 4 April 2020 / Published online: 16 April 2020

(c) The Author(s) 2020

\begin{abstract}
The need for a shift toward healthier and more sustainable diets is evident and is supported by universalized standards for a "planetary health diet" as recommended in the recent EAT-Lancet report. At the same time, differences exist in tastes, preferences and food practices among diverse ethnic groups, which becomes progressively relevant in light of Europe's increasingly multi-ethnic cities. There is a growing tension between current sustainable diets standards and how diverse ethnic resident groups relate to it within their 'culturally appropriate' foodways, raising questions around inclusion. What are dynamics of inclusiveness in migrant food practices? And what does this mean towards the transition to healthy and sustainable food? We study this question among Syrian migrants with different lengths of stay in the Netherlands. Our theoretical framework is based on practice theories, which emphasize the importance of socio-material context and of bodily routines and competences. We use qualitative methods, combining in-depth semi-structured life-history interviews with participant observation. Our findings indicate that inclusiveness takes different forms as migrants' food practices and the food environment change. Regarding health and sustainability in food practices, understandings and competences around particularly fresh food change over time among both short- and long-term migrants, replacing making things from scratch with seasonal products with buying more processed products and out-of-season vegetables and fruits. We conclude that the performances of food practices and their configurations in food environments and lifestyles are dynamic and cannot unequivocally be interpreted as in- or exclusive, but that a more nuanced understanding is required.
\end{abstract}

Keywords Food consumption $\cdot$ Healthy and sustainable food $\cdot$ Food environment $\cdot$ Multi-ethnic city $\cdot$ Migrants · Inclusiveness · Practice theories

\section{Abbreviations}

ST Short-term [migrants]

LT Long-term [migrants]

NGO Non-governmental organisation

Anke Brons

a.brons@aeres.nl

Peter Oosterveer

peter.oosterveer@wur.nl

Sigrid Wertheim-Heck

s.wertheim-heck@aeres.nl

1 Food and Healthy Living, Aeres University of Applied Sciences Almere, Stadhuisstraat 18, Almere 1315 HC, The Netherlands

2 Environmental Policy Group, Wageningen University, Hollandseweg 1, Wageningen 6706 KN, The Netherlands

\section{Introduction}

In light of the serious threats from global climate change and the increasing world population, the need for a shift toward healthier and more sustainable diets is evident (Burlingame and Dernini 2012; Garnett 2014; Lang 2017; EAT-Lancet Commission 2019). Precisely what constitutes a healthy and sustainable diet is, however, a highly debated issue, with new scientific evidence constantly being developed (Tilman and Clark 2014; Nelson et al. 2016; Mason and Lang 2017; Springmann et al. 2018; Béné et al. 2019). A growing body of literature integrates the two aspects of health and sustainability into an overall 'sustainable diet' (Hallström et al. 2018), which Lang (2017) defines as a diet that is healthenhancing, has low environmental impact, is culturally appropriate and economically viable (see also Burlingame and Dernini 2012).

Within this ongoing debate, there is a trend toward universalizing, uniform standards of healthy and sustainable diets that are valid across a nation or even the entire globe. 
The recent EAT-Lancet report on Food, Planet and Health recommends that everyone adopts a 'planetary health diet': a universal diet rich in plant-based, fresh or minimally processed food (EAT-Lancet Commission 2019). On a national level, many countries have a similar approach to integrating health and sustainability into one set of dietary guidelines. In the Netherlands, the Dutch Nutrition Centre promotes the 'Wheel of Five', consisting of five main food groups that make up the recommended plate of an average Dutch consumer, which is based on traditional Dutch foods (Brink et al. 2017). The latest version of the Wheel of Five (2016) for the first time takes into account sustainability, by putting a limit on the amounts of meat and fish recommended per week and advising to consume legumes and nuts.

How can such standardized norms for a sustainable and healthy diet be combined with the central element of 'cultural acceptability' of diets, which requires taking into account dietary tastes and preferences of different cultural groups (Burlingame and Dernini 2012; Lang 2017)? This question is critical in light of the increasingly multi-ethnic cities in Europe (BCFN and MacroGeo 2018; Crul 2016). With a growing diversity in cultural groups moving to cities in larger numbers, there is a rise of so-called 'majorityminority cities' like Amsterdam or Brussels, in which the majority of the urban population consists of cultural minorities, each with their own food practices (Crul 2016).

The required scale of the transformation towards more sustainable and healthy food practices means all citizens need to be on board for it to be effective. Yet, in light of our multi-ethnic societies, generic standards might have limited reach, as they tend to lack cultural sensitivity while food consumption patterns are highly culturally defined (Nicolaou et al. 2009). Additionally, migrants and their home-country food practices may culturally clash with the host culture's definition of what constitutes healthy and sustainable food (Guthman 2008; Johnston et al. 2011; Rice 2015). Moreover, it is crucial to understand cultural acceptability itself as a process rather than as a static goal (Hammelman and Hayes-Conroy 2015). As Hammelman and Hayes-Conroy (2015) argue, the focus of current urban policy is often just on availability of culturally appropriate food, whereas food is much more than just a 'nutrient vessel': it comes with important cultural values and identity. This is particularly relevant in light of increasing calls for more inclusive food system transformations (Bui et al. 2019; Dubbeling et al. 2017; Raja et al. 2017): what exactly does this inclusiveness mean?

This question is also important because much of the current literature on in- and exclusion regarding access to 'good food' focuses primarily on the supply side to explain exclusion, looking at the influence of retail availability and product range on consumption patterns of poor ethnic minorities (Walker et al. 2010). However, as authors such as Alkon et al. (2013), Shannon (2014), Bedore (2014) and more recently also Allcott et al. (2019) argue, the relationship between consumption and provision is not as unidirectional as often thought. Allcott et al. (2019)'s study shows that exposing poorer households to the same products and prices available to higher income households only reduced nutritional inequality by roughly ten percent. Moreover, most literature on in- and exclusion regarding food access is geographically oriented towards the US, where the focus is on black and poor minorities who live in strongly segregated urban environments (Mata 2013; Raja et al. 2008; Walker et al. 2010). These spatial settings generally differ from the European context in their food environments, with a lower prevalence of 'food deserts' and less segregated ethnic neighbourhoods (see for instance Helbich et al. 2017 on Amsterdam).

In short, there is an urgency to understand how inclusiveness regarding healthy and sustainable food works within a multi-ethnic urban context. What are dynamics of inclusiveness in migrant food practices? And what does this mean for the transition toward healthy and sustainable food? In this paper we start by referring to inclusiveness primarily in relation to culture, as being respectful of cultural tastes and preferences (Sustainable Development Goals 2015), further exploring the meaning of this concept through our empirical work.

In answering our research questions, we conducted a comparative analysis between short- and long-term Syrian migrant residents in the Netherlands, investigating how food practices and understandings of health and sustainability develop over time within a changing food environment. We focus on Syrian migrants because this allows for the short-/ long-term migrant comparison as the Netherlands had two Syrian migration flows. One flow dates back several decades and relates to religious persecution of Syrian-orthodox populations in Syria. The other regards the recent influx of Syrians due to the civil war. Within and across these two groups, we study how and to what extent food practices change over the course of migration. We look at how inclusiveness works differently for short- and long-term migrants, also taking into account differences and changes in the food environment and in lifestyles over time. We use a practice theories approach because this is instrumental for uncovering dynamics and arriving at a nuanced understanding of the complexity of inclusiveness.

Below we elaborate on our practices theoretical perspective on inclusiveness and present the conceptual framework of our study. This is followed by an exposition of our methodological approach and a description of the population under study. We then proceed with presenting our empirical results in two main parts, following the main elements of our conceptual model, and end with a discussion and conclusion on our results in light of our research questions. 
Fig. 1 Conceptual framework as inspired by Spaargaren and Van Vliet (2000)

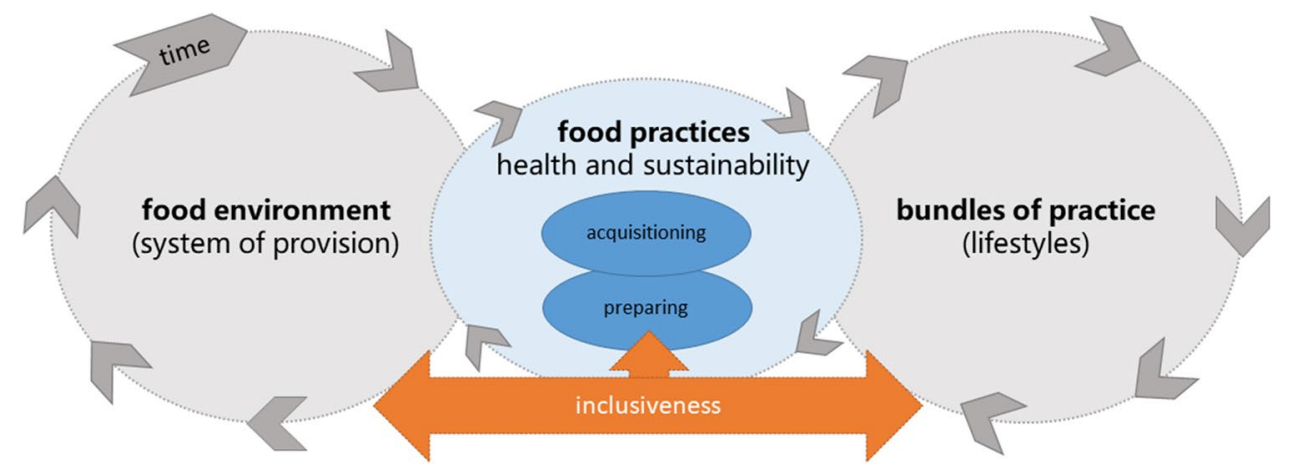

\section{Theoretical and conceptual framework}

In obtaining more nuanced and contextualised understandings of inclusiveness within the dynamics of healthy and sustainable food consumption among short- and long-term (ST-LT) migrant groups, social practice theories that focus on the habitual nature of consumption appear especially suitable. When migrants arrive into a new food environment with existing country-of-origin routines and competences related to food, de- and reroutinization takes place. This dis- and re-embedding means some practices persist, others disappear and new practices may appear. A practice theories perspective highlights these dynamics by examining how food practices are dynamically co-constituted by their material (food) environment and changing lifestyles.

Theories of social practice focus on daily lives by means of identifying routinized behaviour, shared norms, knowledge and competences within a material context (Nicolini 2013). Practice theories aim to bypass both individualist and holistic social ontologies by conceptualizing social reality as made up of 'practices'. Practice theories are an aggregate of theories which emphasize different elements of practice, but key aspects across theorists are embodied routines, skills and knowledge, shared (social) meanings, norms or understandings, and a material infrastructure (Halkier et al. 2011; Schatzki 2002; Shove et al. 2012; Spaargaren and Van Vliet 2000; Warde 2005; Reckwitz 2002).

Studying practices requires the double move of zooming in and out (Nicolini 2013). This two-step approach allows for both a concrete (zoomed-in) and abstract (zoomed-out) understanding of daily practices. Zooming in entails closely examining how practices are actually performed in everyday life, focusing on competences, tastes and preferences and how they may change over time within these food practices. Specifically, we pay attention to people's understandings of health and sustainability.

Subsequently, zooming out means taking a step back, to see how these situated practices relate to other practices in space and over time. In zooming out, practices are studied relationally, comparing and contrasting different instances of the performance of one particular practice over time and space, within the material context of the food environment, as well as in relation to bundles of practice (see Fig. 1). The food environment is defined by Turner et al. (2018) as "the interface that mediates people's food acquisition and consumption within the wider food system" (p. 95) and includes both market-based sources and home growing. This food environment contains many cues and clues for action that inform the performance of food practices, while changing practice elements or changing bundles of practice can in turn also transform the food environment (Warde 2016). Lastly, bundles of practices are sets of practices that are loosely linked based on co-existence in time or space (Schatzki 2011; Shove et al. 2012). Studying bundled practices means understanding how practices connect, either through restricting, enabling or conditioning each other (Shove et al. 2012).

Our conceptual model (see Fig. 1) illustrates how food practices are located in the interaction of the food environment or system of provision with the wider bundles of practices that together constitute daily life. We look at the practices of food acquisitioning and preparing food at home, paying particular attention to meanings or understandings associated with health and sustainability. These food practices are subject to change over time and space, and connect with the food environment and bundles of practices in different ways, corresponding to different degrees of de- and reroutinization among short- and long-term migrants. Sometimes novel practices emerge which link to existing food system practices, while other (elements of) migrant food practices may disappear or transform by integrating with locally dominant practices, as will be illustrated in more detail below.

These processes of forging connections between migrant food practices and locally dominant food habits can take different forms, which have implications regarding their inclusiveness. In considering inclusiveness, we start from the definition of social inclusion by Hinrichs and Kremer (2002) as "an ongoing and reflexive process of full and engaged participation by all interested or affected social actors, regardless of their socio-economic or cultural resources" 
(p. 68). Although we are aware that inclusiveness is a broad concept which is determined by multiple social, economic and cultural factors, in this paper, we understand inclusiveness primarily in relation to the latter aspect of culture, i.e. as being culturally appropriate or acceptable (Hammelman and Hayes-Conroy 2015) or respectful of cultural tastes and preferences (Sustainable Development Goals 2015). We treat inclusiveness as an emerging and dynamic concept and study it inductively, identifying different dynamics of inclusiveness over time and space.

\section{Methodology}

\section{Methods}

Exploring the notions and dynamics of inclusiveness, our study focused on the lived experiences of migrants in their daily food routines and understandings of health and sustainability. Given its exploratory nature, we used qualitative methods to study practices. Given the short-term/ long-term comparative nature of this study we combined in-depth semi-structured life history interviews (accounts of performative action) with food practice observations (direct access to performative action). We applied these methods to study the practices of acquisitioning food and preparing food at home. Within these food practices, we looked at the dynamics in tastes and preferences, and skills and knowledge, in relation to the experiences with the changing food environment. We specifically focused on uncovering (shifting) understandings of health and sustainability.

To inform the interview guide, the study started with the consultation of a dietician from the Arabic region currently residing in the Netherlands. She volunteered with a Dutch NGO called Pharos-a center of expertise that strives to reduce population health disparities and has dedicated programs for migrants. This expert consultation aided an initial understanding of Syrian food culture. Next, the interview guide was tested, after which interviews were conducted to understand current and past performances of the practices of acquisitioning and preparing food. Within the interviews, a life histories approach (Perez 2017) was applied in which people were asked to highlight food-related life events (i.e. favourite childhood food, first time cooking, first meal in the Netherlands) in relation to the two practices under study, to take a historical perspective and understand changing food practices over the course of migration.

The interviews were semi-structured and were conducted between summer 2018 and spring 2019. A total of 26 people were interviewed over a total of 23 sessions (some people were interviewed together, either as couples or friends). Most interviews were conducted in Dutch and on occasion an Arabic translator was used, a Libyan woman who was a native Arab speaker. She was trained by the first author to conduct interviews and did so on a voluntary basis, together with the first author. The interviews were transcribed in Dutch and coded through the open source coding programme QDA Miner Lite. General code categories were drawn up a priori, based on the interview guide, and more specific sub codes were added inductively. Quotes used in this article were translated by the first author who is a native Dutch speaker and have occasionally been edited for grammatical mistakes to ease comprehension. Finally, to get a better understanding of the interaction between food practices and the food environment, the practice of acquisitioning food was observed with five participants in Almere, by accompanying participants in their grocery shopping trips in various stores.

\section{Sampling and recruitment}

Participants were recruited based on their length of stay in the Netherlands, to arrive at a balanced sample of short-term (ST $<5$ years) and long-term (LT $>5$ years) Syrian migrants (see Table 1). Short-term migrants were recruited from the city of Almere, where Syrians have arrived only over the past five years and the food environment is still actively changing. Long-term migrants were recruited from other Dutch cities where there has been a longer presence of Syrians, among which most prominently the city of Enschede. Convenience and snowball sampling was used to recruit participants for both groups, who were approached in various ways. In Almere, the local language education center was approached to recruit participants. A retired volunteer at the Almere asylum seeker center who had a network among the Syrian population was also contacted to recruit participants. The translator was also asked to recruit among her own network among Syrians in Almere. For long-term residents, a primary school in Enschede was approached through a personal contact who put the researchers in touch with mothers from a Dutch language practice class and social hub. Other long-term residents were recruited through the researchers' personal networks.

In general, most Syrians face large differences in socioeconomic status between Syria and the Netherlands, as a recent report on Syrians in the Netherlands outlines (Dagevos et al. 2018). Whereas almost everyone was employed in Syria, currently only $22 \%$ of the Syrians in the Netherlands hold a job, of whom almost half work below their educational level. Roughly a quarter of women used to be employed in Syria, while $42 \%$ indicated managing the household as their primary activity in Syria. Financially, most Syrians struggle to make ends meet in the Netherlands. The large majority of Syrians is religious: $76 \%$ identify as Muslim and $8 \%$ as Christian. In terms of health, the rate of overweight and obesity is significantly higher among 
Table 1 General characteristics of short vs. long term research participants

\begin{tabular}{lll}
\hline & Short-term $(\mathrm{N}=14)$ & Long-term $(\mathrm{N}=12)$ \\
\hline Average length of stay & 3 years (range 10 months-4,5 years) & 20 years (range 5-32 years) \\
Average age & $41(22-63)$ & $39(31-52)$ \\
Average age of arrival in the Netherlands & 38 years old (20-61) & 18 years old (0-47) \\
Origin in Syria & Urban (Damascus, Aleppo, Homs, Qamishli) & 10 rural, 2 urban (all 12 from North-West Syria) \\
Religion & Muslim, Syrian-Orthodox & Syrian-Orthodox \\
Reasons for migrating & Civil war & Religious persecution \\
Place of residence & Almere & Enschede, Zwolle, Rotterdam, Amsterdam \\
Educational level & 5 university & 3 university \\
& 3 vocational school & 4 vocational school \\
Occupation outside the home & 6 high school & 5 high school \\
& 2 employed & 4 employed \\
Housing condition & 2 volunteering job & 1 volunteering job \\
& 5 taking language classes & House (with garden) \\
\hline
\end{tabular}

Syrians than among the average Dutch population, with $26 \%$ of youngsters (ages 15-24) and up to 75\% of people over 45 being diagnosed as overweight (Dagevos et al. 2018).

Regarding our study population, there were some general differences between short- and long-term migrants (see Table 1). In terms of the total study population, 19 were female and 7 were male. All except one was married and had children, varying between babies and adult children who had already moved out or still lived in Syria. Almost everyone had come to the Netherlands with at least some relatives (often parents or in-laws).

\section{Results and analysis}

In this section we present our empirical results by making Nicolini (2013)'s two moves of zooming in and out. We zoom in on the practices of acquisitioning and preparing food, with specific attention to meanings of health and sustainability. Zooming out, we observe how these practices relate to the changing food environment and to changing bundles of practices or lifestyles, and elaborate how these interactions illustrate different dynamics of inclusiveness.

\section{Zooming in: practice as performances}

\section{Acquisitioning food}

Based on our empirical results we identify two types of acquisitioning practices: purchasing (through market-based sources, Turner et al. 2018) and home-growing. Among long-term migrants, food purchasing primarily takes place at regular supermarkets. Ethnic stores, either specifically Syrian or Turkish/Moroccan are also an important source.
Some specific products (milk, cheese and meat) are purchased directly at farms or slaughterhouses. Fresh market shopping is not very prevalent. Participants mentioned the restricted opening times, limited offer and the quality of produce as reasons for not frequenting the Dutch market:

I do not go to the markets very much because the products on the market are not of good quality, especially in terms of freshness. In the Netherlands the market is not really fresh (M, age 45, LT (19yr))

Many long-term migrants are engaged in the practice of home-growing, which is often a continuation of habits from back in Syria, where most people lived in rural areas with gardens:

Almost every house has a vegetable garden, we had one back home and now here. All my sisters-in-law have a garden. We eat fresh, we just pick and eat: Grapes, all kinds of fruit, cucumber, salad, a lot, everything (F, age 31 , LT (8yr))

All long-term migrants in our study live in a house with a garden, which enables them to easily engage in home-growing. Some grow distinct varieties (particularly smaller sized zucchinis and eggplants), as a coping strategy for addressing their needs and preferences for culturally specific food that they could not buy anywhere. Besides more extensive home growing practices, almost all long-term migrants have at least a grapevine in their backyard to be able to make dolma or yaprak. This was almost everyone's favourite dish from childhood and consists of grape leaves filled with rice and vegetables or meat.

By contrast, almost none of the short-term migrants are engaged in home-growing. Only two used to have a garden back in Syria. Material housing conditions of short-term 
migrants also prevent them from growing their own food, as most people live in apartments rather than houses with gardens. Still, only one participant expressed his desire to grow his own vegetables if he would have a garden. As home-growing was not part of the daily routines of most short-term migrants in the past, this practice is not common now either. The regular Dutch supermarket and ethnic store figure prominently in their food purchasing practices. Moreover, the practice of fresh market shopping is integrated into the rhythms of daily life of short-term migrants, where they shop for fruits, vegetables and fish. Back in Syria, it was common to go food shopping almost daily, either at the fresh market or at small shops. These habits are continued in the Netherlands among the recently arrived migrants, many of whom shop almost daily for fresh products. This rhythm of daily shopping also fits well with shopping at ethnic stores, as in ethnic stores the price as well as the quality of fruits and vegetables is lower, meaning these products wither more quickly. This is however less of an issue for these migrants, as they buy fresh fruits and vegetables almost daily.

Among short-term migrants, online resources are also used in acquisitioning practices. This involves finding specific Syrian products through digital networks (Facebook, WhatsApp) in Arabic. ${ }^{1}$ On these online platforms, information is exchanged about where to find a specific kind of Syrian vegetable; what to do with unknown, typically Dutch vegetables; which retail outlet offers the best food quality; or about a new Syrian business in the area. By being available in their native language, these digital tools provide easy access to culturally appropriate food. Both young and old short-term migrants engage in these online platforms, as this married couple notes:

M: 'On Facebook, they always talk, what does that person make, where can you buy that? (...) Someone will say, 'I found a store. It's located in Amsterdam; it sells the small zucchinis. So who wants to go to Amsterdam the next day?' F: 'Yes, usually it is with older people. They cannot get used to life here easily. They still have left their heart and everything in Syria' (M+F, age 32, ST (4.5yr))

This digital coping strategy is not present among longterm migrants, neither young nor old. Some long-term migrants did recall other coping strategies to get specific food items, i.e. asking a relative to bring products from Syria on their travels.

These different coping strategies illustrate the flexibility and creativity of migrants over time in performing their cultural food needs and preferences in their acquisitioning

\footnotetext{
1 The first author got access to the online platforms by looking at them together with the translator.
}

practices. Both short-term and long-migrants generally aim to continue their habits, in which there are some differences among short- and long-term migrants. Adjustment strategies to sustain cultural food practices are accordingly also different among short- and long-term migrants, which is related to changes in the food environment. The recent rise in online shopping means continuing cultural food practices allows for different coping strategies now than when entering the food environment over a decade ago, illustrating the dynamic interaction between food practices and the food environment which will be elaborated below when zooming out. In any case, these differences over time and between groups of migrants illustrate how understanding what is inclusive here and what is not is hard to distinguish from an outsider's view, with migrants sometimes happily taking up their own role in getting what they want and need, and at other times suffering from not being able to eat their preferred cultural food.

\section{Preparing food}

Moving to the practice of preparing food, there are differences in the types of food prepared by short- and long-term migrants. Long-term migrants have gained knowledge about Dutch cuisine and are skilled at cooking typical Dutch dishes, which enables them to regularly prepare these meals. By contrast, short-term migrants are constrained by their lack of competences and know-how about what actually constitutes Dutch cuisine and how to prepare it, although they are curious about it—on several occasions during interviews the first author was asked about Dutch food. Shortterm migrants almost exclusively prepare the Syrian dishes that they are more familiar with, which take significantly more time and skills to prepare.

A recurring theme among all participants is the understanding that fresh is best. Fresh is associated with healthfulness and tastiness, a good and natural rather than a bad and chemical taste, and with being rich in vitamins. Fresh food is also of cultural value: being able to prepare food from scratch is seen as a sign of being a good Syrian woman. For long-term migrants, fresh is associated with home-growing:

You have to eat fresh. I'd never eat a ready-made meal.

I think it's because we grew up with fresh, my father's vegetable garden, we are used to it $(\mathrm{F}$, age 32 , LT (28 yr))

Although the importance of fresh perpetuates over the course of migration, the actual performances around fresh food change upon coming to the Netherlands, among both short- and long-term migrants. In Syria, doing 'fresh' food involved making food from scratch with products from the season that would be stored to last throughout the winter. Fruits and vegetables were bought in bulk (50-100 kg) at 
low prices in the season when it was actually fresh. The practice of preserving was performed by women, who would together engage in canning or drying fruits and vegetables, making tomato paste, all kinds of jam or 'makdous' (stuffed eggplant). The older female research participants were all engaged in this practice back in Syria, and the younger women who migrated before coming of age also recall their mothers and grandmothers doing it in Syria:

If you see the somewhat older Syrian women, they can make so many things. Those jars, the readymade things, they just make it themselves, they make everything themselves (F, age 30, LT (9 yr))

After moving to the Netherlands, fresh remains a central element in participants' food practices but is performed differently. Long-term migrants who engaged in home-growing still consume fresh vegetables from their gardens in summer, but in winter purchase out-of-season vegetables, while those not engaging in home-growing started buying fresh fruits and vegetables year-round regardless of the season. In the Dutch food environment, most fruits and vegetables are always available with much smaller price differences between in and out of season than in Syria. Rather than making from scratch and preserving food, participants now purchase ready-made tomato paste and jam in an ethnic store or a regular supermarket:

R: I always have to have cucumber, fruit and (...) I have to have it. I: And did you eat that in Syria? Could you buy it all year? R: Yes, you can get it. But it's very expensive (F, age 45, LT (28 yr))

So here, all the Syrian women take their habits withthem. So they are also busy making 'makdous'. Butthey stopped doing it here. It's difficult here, becauseeverything is available. Everything is cheap. So whydo I do it and then I am tired. I can just buy it at thesame price (F, age 32, ST (4.5 yr))

This shift towards buying out of season is mostly driven by convenience and financial incentives, and also occurs among the older women interviewed who used to engage in food preserving until recently in Syria, but who do not see the need to continue in the Netherlands as everything is now always available. Consequently, tensions or contradictions between 'doings and sayings' (Schatzki 1996) around fresh food occur. In terms of 'sayings', participants repeatedly emphasize the importance of fresh, seasonal food. Complaints about the taste of out of season vegetables and fruits are also abundant:

Everything comes from the fridge. Nothing is fresh. (...) For example there are the cucumbers, I saw them in Emmen [a city in the Netherlands], all in greenhouses. And if you get these big cucumbers or these tomatoes and peppers and they all grow so quickly, well, then you know that it's not really fresh, that it doesn't grow by itself. It's all pumped up with needles, with water (M, age 35, LT (30 yr))

However, in terms of actual 'doings', this participant and others still buy these cucumbers and tomatoes all year long, regardless of the season. Convenience seems to trump convictions and taste in the new food environment where everything is always available.

There are two exceptions to changing performances of practices around fresh, which involve the products of grape leaves and labneh (a kind of strained yoghurt or fresh cheese). As mentioned before, grape leaves are essential for preparing the popular dish of dolma or yaprak. However, some long-term migrants note that fresh grape leaves were hard if not impossible to buy in the Netherlands, so many resorted to drying the grape leaves from their backyard grapevines in order to be able to also make yaprak in winter. By contrast, neither growing nor drying grape leaves is common practice among short-term residents, who instead buy dried grape leaves at ethnic stores.

The second example concerns the practice of making labneh. This practice perpetuates across migration among some, both long- and short-term, older and younger practitioners, although they are engaged in it for different reasons. Two relatively young interviewees (ages 34/35, ST (3.5/4 years) were recruited to the practice only upon coming to the Netherlands. They enrolled because they could not find the labneh they wanted in the existing food environment (similar to the grape leaves). They acquired the skills and competences to make labneh online, through Facebook and YouTube. However, this performance of the practice disappeared as soon as it had to compete with another, new means of acquiring labneh (i.e. online shopping), which was more convenient. This practice enrolment and engagement differs from other both long- and short-term migrants who already learned the required skills from their mothers when growing up, either in the Netherlands or in Syria, and are in the habit of doing it from a younger age. This example illustrates how the performances of a practice may look similar but that meanings, recruitment and engagement within it can take different forms, contributing to different dynamics of inclusiveness.

\section{Health and sustainability}

Within the practices of acquisitioning and preparing food, we zoom in further on understandings of health and sustainability, and their possible change over the course of migration. In being de-routinized upon coming to a new country with a different food environment and food habits, interviewees had often started to reflect more on their own food 
practices, with changing ideas about health and about the healthfulness of Syrian cuisine. In explaining what meanings they associate with health, many participants describe health in terms of what a healthy diet should not contain: a healthy diet consists of less fat, less sugar and less salt. This is often referred to as the opposite of the Syrian cuisine, which contains a lot of sugar and animal fat (in particular ghee):

I don't think Syrian food is healthy. No, we use a lot of fat. Sometimes they make salad with olive oil, then it's OK, but most use butter very often, or ghee. And that has a lot of fat in it, and that's really not healthy I think. Because my mother also has issues with her cholesterol, and the doctor says, there's so much fat in Syrian food, take it easy and don't eat that too much (F, age 34, LT (born in NL))

This focus on what is not healthy and specifically participants' reflexivity towards Syrian cuisine came about in different ways by cues in the socio-material environment, either back in Syria or in the Netherlands. Some interviewees recall Syrian information campaigns on television in recent years on reducing fat and oil consumption. Yet, most had started reflecting on health in relation to their food practices after coming to the Netherlands. As one interviewee (F, age 32, ST (4.5 years)) notes, "Whereas in Syria it was my own choice to eat healthily or just meat and rice, in the Netherlands you read and hear about healthy food all the time".

In response, the family's food practices shifted to consuming more vegetables and fruits, less sugar and to having more diversity in their meals. The dietician also notes changing performances around health, illustrating this with a traditional Arabic dish called 'musakhan' that is typically served with Arabic flatbread which contains a lot of fat. Now, instead of the Turkish bread with all the oils and fats, she notes that people start using thin bread, tortilla etc."musakhan 2018". Others became aware of food-related health issues through personal experience, and recall changing their food practices (towards less fat, less sugar and less salt) after going to the doctor in the Netherlands for obesity, type 2 diabetes or another food-related health issue.

Sometimes there are explicit tensions between Dutch dietary guidelines and cultural food practices, leading to challenges for migrants. For instance, one interviewee (F, age 33, LT (20 years)) recalls that when she was pregnant and suffered from iron deficiency, the doctor prescribed her to eat more rye bread and apple spread, which is typically Dutch food. This required some cultural know-how and competences that this interviewee did not possess at the time, which was constraining for her. She felt a mismatch between the doctor's advice and her own food habits: "And at the beginning I was, how I do that because I am used to eating something different at home. (...) And she told me, eat that and eat that. And yeah, it's very difficult to eat differently".
Recognizing these constraints experienced by migrants in receiving food-related health recommendations, the dietician indicates that the NGO she volunteered with was actually in the process of releasing an Arabic version of the Wheel of Five, which indicates a kind of mutual reflexivity. In this version, the NGO will translate not only the texts but also the kinds of foods included in the guidelines into culturally appropriate foods, in light of the current discrepancies with the food practices of the many different ethnicities of refugees and migrants they work with. This serves as one empirical example of how healthy and sustainable dietary guidelines could become more inclusive by taking into account more culturally acceptable foods.

Another dominant understanding of health among participants is related to consuming fruits and vegetables. Some refer to the Syrian cuisine as rather healthy because it commonly includes fresh vegetables at all meals as well as many fruits. The need to incorporate sufficient amounts of vegetables into the diet is particularly brought about by having children. This life event sparks an increased motivation for eating more vegetables among many participants. The issue of sugar is also raised in relation to children as well as in a broader sense, where the Dutch food environment is sometimes blamed for its wide availability of processed sugary foods:

These days you really have to pay attention to those things. (...) There's sugar in everything. And in Syria, three quarters of the food came from the land. Candy doesn't come from a tree, I always say. It's all natural wat you consume there. And here it isn't (F, age 33, LT (20 yr))

Many others do however note that traditional Syrian cuisine includes many sugary snacks such as baklava, which also poses health risks.

Moving to understandings of sustainability, the concept of sustainability is hard to translate in Arabic: the equivalent is not commonly known among participants. Only one of the short-term migrants knows the meaning of the word itself, because she took Dutch language classes-but only in relation to mobility, not food. Among long-term migrants, the term is recognized more frequently but does not figure in their daily food practices. Two participants associate sustainability with eating seasonal food. However, although there is much awareness, know-how and appreciation of consuming seasonal food, it does not figure strongly in their current food practices after migration.

Like seasonality, there are more aspects within interviewees' food practices that could be earmarked as sustainable, when relying on the abovementioned current guidelines for sustainable consumption practices (EAT-Lancet Commission 2019; Brink et al. 2017). Such practices or practice elements are 'inconspicuously sustainable': not intentionally 
sustainable but nonetheless having positive environmental effects (following Dubuisson-Quellier and Gojard 2016). For instance, religious fasting was common among many longterm migrants who were Syrian-Orthodox, which entailed consuming no land animal products on Wednesdays and Fridays nor during the 40 days of Lent before Easter-essentially eating a plant-based diet for two days a week. Consuming local food is another example: back in Syria, in particular long-term migrants who left more than a decade ago were in the habit of acquisitioning only locally produced food (i.e. produced in Syria) in stores and markets, or engaged in home growing. All of these routines and relationships with food (being connected to local and seasonal food consumption and/or production and having a plant-based diet) are an integral part of the cultural identity of these migrants but are also significant in terms of sustainability. These habits continue only among some long-term migrants after migration, by home-growing and shopping at local farms and slaughterhouses. In short, the process of migration changes practical understandings of health and sustainability as well as cultural relationships with food, with increased reflexivity in terms of health sometimes leading to healthier food practices, but potentially sustainable practices often change towards less sustainable practices over the course of migration.

\section{Zooming out}

\section{Changing food practices, lifestyles and bundles of practices}

In zooming out, we first look at how changes in food practices can happen through changing lifestyles or bundles of practices. Upon migration, many daily practices become deroutinized, as one interviewee who volunteers to help out newly arrived Syrians notes:

The rhythm, (...) they really miss the system. They cannot live systematically, with everything being a routine. So for instance, having to wake up early in the morning, school, children, making appointments, being on time, they are not used to that (M, age 44, LT (18 yr))

This de- and re-routinization also (in)directly affects food practices, as "common patterns of adjustment reported [are] often a result of changes in practices other than those directly associated with eating" (Warde 2016, p. 133/4). We illustrate this by highlighting how within changing occupational household dynamics three practices bundle with and change food practices: working outside the home, caring for children or parents and going to school.

First, through enrolment in the practice of working, changes occur within eating practices. When participants start working, this almost immediately affects meal timing among both short- and long-term migrants. In Syria, the main meal took place between two and three in the afternoon, and a light dinner (similar to breakfast) was consumed around 7 or 8 . In the Netherlands, as soon as one adult in the household starts working, these times shift to having the main hot meal around 5 p.m. or 6 p.m., as is common practice in the Netherlands: "I have to eat like Dutch people because I go to school, I work. The break time is like Dutch people so you have to change" (F, age 33, LT (20 years)). By bundling with working in this way, the temporality of eating practices thus changes and adapts more to local practice rhythms. This contrasts with households in which no one works, where meal timing continues according to Syrian rhythms, also among long-term migrants.

The practice of working also bundles with the practice of food preparing, marked by competition for the same resource: time. These practices are connected through changing gender roles which influence the temporality of both practices. Upon coming to the Netherlands, some women start to work or work at different times than in Syria. This constrains them by limiting their available time for cooking, leaving less time for the typically elaborate Syrian dishes and for cooking from scratch:

Because my mother had more time at home, I work and before that I was studying and it's not like I always have a lot of time to make a big meal like Syrians (...). They cook a big meal almost every day. I can't do that every day. It's more like in the evening some soup, spaghetti, macaroni, some easier meals (F, age 33 , LT (20 yr))

Some women now only prepare more elaborate Syrian dishes in the weekend, when they frequently have family and friends over for breakfast or lunch, as was common in Syria. Participants also express that caring for family (children or elderly parents) takes up more time in the Netherlands and similarly limits their time for food preparing.

Finally, enrolment in the practice of going to school leads to adjustments in the practice of eating, in terms of meal timing and meal content. Dutch eating routines at schools consist of bringing sandwiches to school which are consumed around noon. Adapting to these new practice rhythms is challenging at first for many Syrians. One interviewee (M, age 44, LT (18 years)) noted that he often hears about Syrian children not bringing sandwiches to school for lunch, as they are still in the habit of eating after school, around 3 p.m. However, after a while, Syrian children also start bringing sandwiches to school. This habit in turn influences eating practices at home by introducing Dutch bread to the breakfast, which is common even among short-term migrants who had an otherwise predominantly Syrian diet. In short, through changing lifestyles and enrolment into locally common practices with their rhythms, competences, materials 
and meanings, the performance of cultural food practices is sometimes constrained and sometimes enabled, with cultural identity changing concurrently. This illustrates how inclusiveness itself is dynamic, whereby what is culturally acceptable or not is changing over time and varies between different people and practices.

\section{Changing food practices in a changing food environment}

Migrant food practices are not only affected by changing lifestyles but are also embedded within a changing food environment. Building on the food-based life histories, changes in the food environment became apparent when comparing experiences of long- and short-term migrants. This goes both for the Syrian food environment migrants left and for the Dutch food environment they entered. When long-term migrants left, there was little 'multicultural' or 'globalised' food such as kiwis on offer in Syria, which was more common when short-term migrants left Syria. Moreover, the country was not at war yet when long-term migrants left, and the availability of food was not an issue, while for some short-term migrants, buying sufficient food in the Syrian food environment was a challenge during the war. This translated into different expectations of the food environment upon migration: some short-term migrants just aim for having enough food, which is more important than for instance consuming healthy food.

When the first long-term migrants we interviewed arrived in the Netherlands around thirty years ago, the offer of ethnic food was also limited. Ethnic stores in the Netherlands did exist but were Turkish or Moroccan rather than Syrian, and were not as omnipresent as today, which rendered the process of acquiring culturally appropriate food more challenging (see also Huizinga and van Hoven 2018). Over the past decade or so, ethnic food entered the mainstream food system, with Dutch supermarkets increasingly offering ethnic, including Syrian, food products:

Now everything can be found. After the arrival of the Syrian migrants, the goods are everywhere. They can even deliver Syrian food to your home for free. Before there wasn't pomegranate syrup or tahini. Now if you need them you can call and it arrives within 24 hours. When I arrived here in 1992, it was not there because there was no demand. Eggplants were not there, basil, nobody knew it (M, age 45, LT (26yr))

With the increase in ethnic food products on supermarket shelves, short-term migrants face a different food environment than long-term migrants did in their days upon migration. This corresponds with different practice dynamics, rhythms and adaptation strategies among longand short-term migrants in interaction with the changing food environment. For instance, one interviewee recalls having to drive $15 \mathrm{~km}$ to the next Turkish store:

We went once every two months or so, but now I can find it everywhere. I told the new people, you have a lot more luck, you can find it anytime you want. You can even see it on Facebook (F, age 33, LT (20 yr))

This example illustrates how the food environment is actively changing, in a relationship of co-creation with migrant food practices. In Enschede, where there has been a continuous and growing community of Syrian migrants since at least two decades, there are multiple Syrian shops which have been present for over a decade. By contrast, in Almere with its relatively new Syrian community, the first Syrian store appeared only around two or three years ago, although many Turkish and Moroccan ethnic stores had been present in the food environment. The Almere food environment is also still actively changing, with for instance a new Syrian bakery opening during the interviewing period.

This interaction between food environment and migrant food practices demonstrates a variety in practice configurations of emergence, integration and transformation over different times and places, illustrating once more how cultural inclusiveness is dynamic rather than static as both the food environment and food practices change. We will illustrate this again through the case of labneh. In Enschede, Syrian migrants who wanted to make labneh started going to Dutch farmers to buy milk, who gradually ended up selling labneh themselves. Here, ethnic food was integrated into an existing local food provisioning practice, ran by Dutch entrepreneurs, implying a transformation of an existing practice. By contrast, in Almere a novel, separate practice emerged to provide for labneh: businesses initiated and run by Syrian migrants themselves, with labneh being ordered online and home-delivered. Similarly, for meat, participants from Almere shopped at ethnic stores which offer halal meat. In Enschede, participants instead went to the Dutch butcher who possessed the required skills to prepare meat for typically Syrian dishes such as kibbeh:

I order meat from the butcher. I will say, 2 kilos for kibbeh, 3 kilos for kebab. He knows. Yes, sometimes he asks, for kibbeh or for kebab? (...) He definitely doesn't know the taste, but he knows the name (F, age 31, LT (8 yr))

These changing food environments indicate that there is interaction between food environment and food practices, and that this interaction does not evolve in the same way over time and space, indicating diversity between different practices and people also in terms of what might be inclusive or not. 


\section{Dynamics of inclusiveness in relation to health and sustainability}

As our results indicate, food practices, their performances and configurations in food environments and lifestyles are dynamic and cannot unequivocally be interpreted as in- or exclusive. Returning to our understanding of inclusiveness as "full and reflexive participation" from Hinrichs and Kremer (2002) while being respectful of cultural dietary needs and preferences, our findings illustrate how this concept of inclusiveness can take different forms within specific contexts. As performances of practices within a food environment change over time and space, with an older versus a newer migrant community, so do the dynamics around inclusiveness change. We identify three main ways in which these dynamics work, which coincide with the three central elements of the conceptual model presented in Fig. 1: (1) the interaction between food practices and lifestyles or bundles of practices is dynamic; (2) performativity of food practices is dynamic; and (3) the interaction between food practices and the food environment is dynamic.

First, lifestyles or bundles of practices are not static. Changes in geographic and occupational household dynamics contribute to changes in cultural identity and related food practices. Dietary tastes and preferences change over the course of migration, which can be characterized by a spectrum running from maintaining one's cultural identity (multiculturalism) to adapting to the local cultural habits (homogenization), with many hybrid forms in between. Change along the spectrum does not always happen voluntarily: participation in some practices dictates the rhythm or content of others bundled to it. This makes it difficult to deem one particular practice or either end of the spectrum more or less inclusive than the other. For instance, by going to school, know-how about Dutch food habits increased and practices adapted accordingly to include Dutch bread into breakfast. At the same time, by starting to work, meal times had to change towards local practice rhythms. Both are examples of shifts toward cultural homogenization, but they are not necessarily similarly in- or exclusive. This also underlines the need to understand the cultural acceptability of food as a dynamic process rather than as static, as previously argued by Hammelman and Hayes-Conroy (2015).

Secondly, food practices and their performances change. Sometimes new practices emerged that became linked to existing food system practices (e.g. Syrian migrants starting a Syrian cheese business). Other times new practice elements were integrated into existing local provisioning practices (a Dutch farmer including Syrian labneh in their offer), thereby transforming local food system practices. Additionally, for migrants, acquiring food according to cultural tastes and preferences in a new food environment sometimes meant enrolment into new practices, as happened in the case of home growing, food preserving and online shopping practices. However, although the performance of some of these practices may look similar from the outside, they contained different meanings and modes of engagements. In the case of making labneh, some practitioners performed this practice because they were already used to doing it, whereas others started because they experienced a lack of availability of labneh in their new food environment. Unravelling these dynamics of practice shows how complex the issue of inclusiveness is: simply deeming the practice of making labneh as such to be in- or exclusive is difficult and does not do justice to the complexity of people's experiences.

Thirdly, food practices also have an interactive relationship with the food environment. Migrant food practices influence the food environment and vice versa, which works out differently in different times and spaces. Short-term migrants arriving into the current food environment that offers opportunities for digital communities and platforms develop different coping strategies to fulfil their cultural dietary needs and preferences than long-term migrants did back in their day, which translates into different practices.

Finally, relating these dynamics of inclusiveness to health and sustainability, we identify potential for latching onto existing elements of migrant food practices for transitioning towards a healthier and more sustainable food system. In terms of health, being de-routinized after migration stimulates reflection, foregrounding (unhealthy) elements of people's food practices, which has potential for making practices healthier. Furthermore, a tool like the Arabic food groupsbased Wheel of Five encourages diversity in dietary standards, providing a better match with migrant food practices and making healthy food recommendations more culturally appropriate.

Regarding sustainability, the identified know-how on fresh, seasonal and local food among the migrants studied offers potential for the transition towards a more sustainable food system through a focus on such fresh, local and seasonal food. This focus fits well with some elements of migrant food practices, who are routinized in buying such food. Consuming — and to some extent also growing — local, seasonal and fresh food is thus essentially part of their cultural identity, and this cultural knowledge should be appreciated (Hammelman and Hayes-Conroy 2015). Instead of losing these cultural habits upon migration, these routines should be facilitated and encouraged - although perhaps not explicitly in the name of sustainability, as this framing did not resonate. Rather, a meticulous approach is needed to make the connection between sustainability and migrants' everyday food practices. For instance, as many migrants frequently shop at ethnic stores and/or fresh markets, offering more fresh, local and seasonal food that is also culturally acceptable in these places could be an interesting option for stimulating healthy and sustainable consumption. 


\section{Discussion and conclusion}

This paper aimed to understand what inclusiveness means in light of our increasingly multi-ethnic cities. Appeals for a more inclusive food system suggest a current state of exclusion for vulnerable groups like migrants. However, our results indicate that rather than working from a normative frame that is imposed top-down on a given population, a more nuanced understanding of what constitutes in- and exclusion is required. Inclusiveness is a dynamic process, in which migrants can be capable of including themselves, demonstrating creativity in sustaining their cultural practices and developing coping strategies in interaction with a changing food environment. Our practice theories approach has been instrumental in identifying these dynamics. We see a number of ways in which our findings can contribute to shaping more robust pathways to a healthier and more sustainable food system that is also inclusive: (1) moving beyond a supply side only-perspective on in/exclusion; (2) taking a critical look at nationally or even globally defined dietary guidelines; (3) emphasizing citizens' creativity in organizing their food practices; (4) acknowledging that migrants are also consumers driven by 'lifestyle' needs like convenience; and (5) identifying health as an interesting access point for dietary change. At the core of these recommendations lies the observation that inclusive transitions to sustainable diets should be informed by how migrants actually engage with food in their daily lives-rather than making assumptions about their food habits and values from a distance-and that these food practices are dynamic and change over time and space.

First of all, in response to food desert thinking we referred to in the introduction, our findings highlight a more multidirectional and dynamic interaction between food environment and food practices. We fully support Hammelman and Hayes-Conroy (2015)'s call to look beyond food availability only, and start paying attention to "how cultural acceptability develops through complex relationships between people and food systems" (p. 44) in order to effectively understand cultural inclusiveness. Food culture, practices and the food environment are dynamic in multiple senses, with changes occurring in both the country of residence and country of origin. This requires appreciating the complexity of everyday life of how migrants negotiate their food practices, as our study has aimed to illustrate through a practice theories lens.

Secondly, in line with this complexity of daily life, dynamics and variation between groups of migrants, our findings illustrate how there are limits to the extent to which nationally defined dietary guidelines can be effective. For instance, as our findings demonstrated, seasonal food consumption may be prevalent in the home country but is affected and diminished by migrating to a new food environment. Even though migrants' 'home-country' practices of seasonal consumption might fit with the Dutch dietary guidelines in terms of consuming fresh foods, the specific foods associated with home-country seasonal consumption are not necessarily available as such in the Netherlands. Drawing up one set of guidelines that is culturally appropriate or inclusive is therefore complicated. Rather, adaptive and reflexive capacity is key, where migrants themselves are being involved, for instance following the initiative of the NGO Pharos to draw up an Arabic foodsbased Wheel of Five in cooperation with health experts and consumers from the region.

Thirdly, for local governments working on inclusive food system transformations, our research suggests that policymakers should recognize citizens' flexibility and creativity when tailoring interventions. Inclusiveness is hard to measure based on simple socio-economic parameters but is rather diverse in form and subject to change over time and space. Migrants themselves are not passively waiting to be included but actively shape their food environment and develop creative coping strategies, as "knowledgeable and capable agents" (Giddens 1984), with some transformative capacity to actively interact with and change elements in the food environment to fulfil their dietary needs and preferences. Rather than using quantitative parameters such as only measuring the availability of certain "culturally appropriate" food items to indicate in-or exclusion, our qualitative approach shows the complexity of what inclusiveness means in practice, illustrating different dynamics between shortand long-term migrants and in interaction with changing food environments and bundles of practices.

Fourthly, convenience also played a role in the changing lifestyles and food practices of migrants. Inclusiveness means taking into account that also migrants seek convenience, in acquiring and preparing foods to their 'culturally appropriate' foods, which was illustrated by the example of preparing or buying labneh. This means that solutions such as proposing urban agriculture for cultural inclusion might not be appropriate when migrants' changing lifestyles increasingly require convenience. The supposition of migrants having the time and interest needed to grow their own food, practiced by many short-term migrants, might not uphold when lifestyles change, like women working out-ofhome. This research only lightly touched upon convenience and further research into the role of convenience in relation to inclusiveness is required, which might differ between groups of people.

Finally, when defining an inclusive food system from a health and sustainability perspective, our findings indicate that health is an easier access point than sustainability. Deand reroutinization upon migration often includes moments 
of reflection that help transition to healthier food practices. For sustainability, the connection with migrant food practices is less obvious and more attention should be paid to how sustainability can be integrated within food practices. Moreover, sometimes there were trade-offs, as in the case of fresh vegetables, where migrants changed towards buying fresh year-round rather than eating preserved food, which was beneficial in terms of health but less so in terms of sustainability.

The present study has zoomed in on one particular group of migrants to conduct a comparison over time, among different lengths of stay. This allowed for an in-depth understanding of the dynamics of inclusiveness and change among this population group. While there are most likely similarities to be found in migrant groups from the same region with similarities in food culture, our sample also shows how diverse the dynamics of inclusiveness already are within one cultural group over time. In reflecting upon the cultural diversity of most current metropolises, further research is therefore required among other cultural groups, to explore to what extent the identified dynamics transpire among other migrant populations. This calls for a careful consideration of cultural food practices among different groups of migrants in a city in order to achieve truly inclusive strategies to feeding the multi-ethnic city.

Acknowledgements This research has been funded by Gemeente Almere (Almere 2.0) in cooperation with Flevo Campus. We would like to thank all participants who shared their stories with us and the editor and three anonymous reviewers for their constructive and helpful comments on earlier versions of the paper.

Author contributions All authors conceived and designed the experiments; the first author collected and analyzed the data; and all authors wrote the paper.

Open Access This article is licensed under a Creative Commons Attribution 4.0 International License, which permits use, sharing, adaptation, distribution and reproduction in any medium or format, as long as you give appropriate credit to the original author(s) and the source, provide a link to the Creative Commons licence, and indicate if changes were made. The images or other third party material in this article are included in the article's Creative Commons licence, unless indicated otherwise in a credit line to the material. If material is not included in the article's Creative Commons licence and your intended use is not permitted by statutory regulation or exceeds the permitted use, you will need to obtain permission directly from the copyright holder. To view a copy of this licence, visit http://creativecommons.org/licenses/by/4.0/.

\section{References}

Alkon, A.H., D. Block, K. Moore, C. Gillis, N. DiNuccio, and N. Chavez. 2013. Foodways of the urban poor. Geoforum 48: 126-135.

Allcott, H., R. Diamond, J.P. Dubé, J. Handbury, I. Rahkovsky, and M. Schnell. 2019. Food deserts and the causes of nutritional inequality. The Quarterly Journal of Economics 134 (4): 1793-1844.

BCFN and MacroGeo. 2018. Food \& migration: Understanding the geopolitical nexus in the Euro-Mediterranean. BCFN and MacroGeo. https://paper.foodandmigration.com. Accessed 23 Mar 2020.

Bedore, M. 2014. Food desertification: Situating choice and class relations within an urban political economy of declining food access. Studies in Social Justice 8 (2): 207-228.

Béné, C., P. Oosterveer, L. Lamotte, I.D. Brouwer, S. de Haan, S.D. Prager, E.F. Talsma, and C.K. Khoury. 2019. When food systems meet sustainability-Current narratives and implications for actions. World Development 113: 116-130.

Brink, E.J., A. Postma-Smeets, A. Stafleu, and D. Wolvers. 2017. The wheel of five factsheet. Voedingscentrum website. https://mobie 1.voedingscentrum.n1/Assets/Uploads/voedingscentrum/Docum ents/Professionals/Pers/Factsheets/English/Fact\%20sheet\%20The \%20Wheel\%20of\%20Five.pdf. Accessed 23 Mar 2020.

Bui, S., I. Costa, O. De Schutter, T. Dedeurwaerdere, M. Hudon, and M. Feyereisen. 2019. Systemic ethics and inclusive governance: two key prerequisites for sustainability transitions of agri-food systems. Agriculture and Human Values 36 (2): 277-288.

Burlingame, B., and Dernini, S. 2012. Sustainable diets and biodiversity: Directions and solutions for policy, research and action. Food and Agriculture Organization of the United Nations (FAO) website. https://www.fao.org/3/i3004e/i3004e.pdf. Accessed 23 Mar 2020.

Crul, M. 2016. Super-diversity vs. assimilation: how complex diversity in majority-minority cities challenges the assumptions of assimilation. Journal of Ethnic and Migration Studies 42 (1): 54-68.

Dagevos, J., W. Huijnk, M. Maliepaard, and E. Miltenburg. 2018. Syriërs in Nederland. Sociaal Cultureel Planbureau website. https ://www.scp.nl/dsresource?objectid=7ad7406c-723d-4954-a601239b4ae10008\&type=org. Accessed 23 Mar 2020.

Dubbeling, M., G. Santini, H. Renting, M. Taguchi, L. Lançon, J. Zuluaga, L. de Paoli, A. Rodriguez, and V. Andino. 2017. Assessing and planning sustainable city region food systems: insights from two Latin American cities. Sustainability 9 (8): 1455.

Dubuisson-Quellier, S., and S. Gojard. 2016. Why are food practices not (more) environmentally friendly in France? The role of collective standards and symbolic boundaries in food practices. Environmental Policy and Governance 26 (2): 89-100.

EAT-Lancet Commission. 2019. Food, planet, health: Healthy diets from sustainable food systems. The Lancet website. https://www. thelancet.com/journals/lancet/article/PIIS0140-6736(18)31788-4/ fulltext. Accessed 23 Mar 2020.

Garnett, T. 2014. Changing what we eat: A call for research \& action on widespread adoption of sustainable healthy eating. Food Climate Research Network website. https://www.fcrn.org.uk/sites/defau 1t/files/fcrn_wellcome_gfs_changing_consumption_report_final .pdf. Accessed 23 Mar 2020.

Giddens, A. 1984. The constitution of society: Outline of the theory of structuration. Berkeley: University of California Press.

Guthman, J. 2008. 'If they only knew': Color blindness and universalism in California alternative food institutions. The Professional Geographer 60 (3): 387-397.

Halkier, B., T. Katz-Gerro, and L. Martens. 2011. Applying practice theory to the study of consumption: Theoretical and methodological considerations. Journal of Consumer Culture 11 (1): 3-13.

Hallström, E., J. Davis, A. Woodhouse, and U. Sonesson. 2018. Using dietary quality scores to assess sustainability of food products and human diets: A systematic review. Ecological Indicators 93: 219-230.

Hammelman, C., and A. Hayes-Conroy. 2015. Understanding cultural acceptability for urban food policy. Journal of Planning Literature 30 (1): $37-48$. 
Helbich, M., B. Schadenberg, J. Hagenauer, and M. Poelman. 2017. Food deserts? Healthy food access in Amsterdam. Applied Geography 83: 1-12.

Hinrichs, C., and K.S. Kremer. 2002. Social inclusion in a Midwest local food system project. Journal of Poverty 6 (1): 65-90.

Huizinga, R.P., and B. van Hoven. 2018. Everyday geographies of belonging: Syrian refugee experiences in the Northern Netherlands. Geoforum 96: 309-317.

Johnston, J., M. Szabo, and A. Rodney. 2011. Good food, good people: Understanding the cultural repertoire of ethical eating. Journal of Consumer Culture 11 (3): 293-318.

Lang, T. 2017. Re-fashioning food systems with sustainable diet guidelines: Towards a SDG2 strategy. Food Research Collaboration website. https://foodresearch.org.uk/publications/re-fashioning -food-systems-with-sustainable-diet-guidelines/. Accessed 23 Mar 2020.

Mason, P., and T. Lang. 2017. Sustainable diets: How ecological nutrition can transform consumption and the food system. London: Routledge, Taylor \& Francis Group.

Mata, C.T. 2013. Marginalizing access to the sustainable food system: An examination of Oakland's minority districts. Lanham, MD: University Press of America.

Nelson, M.E., M.W. Hamm, F.B. Hu, S.A. Abrams, and T.S. Griffin. 2016. Alignment of healthy dietary patterns and environmental sustainability: A systematic review. Advances in Nutrition 7 (6): $1005-1025$.

Nicolaou, M., C.M. Doak, R.M. van Dam, J. Brug, K. Stronks, and J.C. Seidell. 2009. Cultural and social influences on food consumption in Dutch residents of Turkish and Moroccan origin: A qualitative study. Journal of Nutrition Education and Behavior 41 (4): 232-241.

Nicolini, D. 2013. Practice theory, work, and organization: An introduction. Oxford: Oxford University Press.

Perez, R.L. 2017. Interviewing epistemologies: From life history to kitchen table ethnography. In Food culture: Anthropology, linguistics, and food studies, ed. J. Chrzan and J. Brett, vol. 2, 47-57. New York: Berghahn.2

Raja, S., C. Ma, and P. Yadav. 2008. Beyond food deserts: Measuring and mapping racial disparities in neighborhood food environments. Journal of Planning Education and Research 27 (4): 469-482.

Raja, S., K. Morgan, and E. Hall. 2017. Planning for equitable urban and regional food systems. Built Environment 43 (3): 309-314.

Reckwitz, A. 2002. Toward a theory of social practices: A development in culturalist theorizing. European Journal of Social Theory 5 (2): 243-263.

Rice, J.S. 2015. Privilege and exclusion at the farmers market: Findings from a survey of shoppers. Agriculture and Human Values 32 (1): 21-29.

Schatzki, T.R. 1996. Social practices: A Wittgensteinian approach to human activity and the social. Cambridge, UK: Cambridge University Press.

Schatzki, T.R. 2002. The site of the social: A philosophical account of the constitution of social life and change. University Park, PA: Pennsylvania State University Press.

Schatzki, T.R. 2011. Where the action is (on large social phenomena such as sociotechnical regimes). Working Paper 1, Sustainable Practices Research Group.

Shannon, J. 2014. Food deserts: Governing obesity in the neoliberal city. Progress in Human Geography 38 (2): 248-266.
Shove, E., M. Pantzar, and M. Watson. 2012. The dynamics of social practice: Everyday life and how it changes. Thousand Oaks, CA: Sage.

Spaargaren, G., and B. Van Vliet. 2000. Lifestyles, consumption and the environment: The ecological modernization of domestic consumption. Environmental Politics 9 (1): 50-76.

Springmann, M., M. Clark, D. Mason-D'Croz, K. Wiebe, B.L. Bodirsky, L. Lassaletta, W. de Vries, S.J. Vermeulen, M. Herrero, K.M. Carlson, M. Jonell, M. Troell, F. DeClerck, L.J. Gordon, R. Zurayk, P. Scarborough, M. Rayner, B. Loken, J. Fanzo, H.C.J. Godfray, D. Tilman, J. Rockström, and W. Willett. 2018. Options for keeping the food system within environmental limits. Nature 562 (7728): 519-525.

Sustainable Development Goals. 2015. Transforming our world: The 2030 agenda for sustainable development. Sustainable Development Goals Knowledge Platform. https://sustainabledevelopme nt.un.org/post2015/transformingourworld. Accessed 23 Mar 2020.

Tilman, D., and M. Clark. 2014. Global diets link environmental sustainability and human health. Nature 515 (7528): 518-522.

Turner, C., A. Aggarwal, H. Walls, A. Herforth, A. Drewnowski, J. Coates, S. Kalamatianou, and S. Kadiyala. 2018. Concepts and critical perspectives for food environment research: A global framework with implications for action in low-and middle-income countries. Glob Food Secur 18: 93-101.

Walker, R.E., C.R. Keane, and J.G. Burke. 2010. Disparities and access to healthy food in the United States: A review of food deserts literature. Health \& Place 16 (5): 876-884.

Warde, A. 2005. Consumption and theories of practice. Journal of Consumer Culture 5 (2): 131-153.

Warde, A. 2016. The practice of eating. Cambridge, UK: Polity Press.

Publisher's Note Springer Nature remains neutral with regard to jurisdictional claims in published maps and institutional affiliations.

Anke Brons is a Ph.D. candidate at Aeres University of Applied Sciences and at the Environmental Policy Group at Wageningen University, both in the Netherlands. Her Ph.D. project focuses on questions of inclusiveness around access to healthy and sustainable food in a Western urban context, from a sociological perspective. Her research interests include food consumption, food systems, social equity and social practice theories.

Peter Oosterveer is a professor at the Environmental Policy Group at Wageningen University, the Netherlands. His research interests are in global public and private food governance arrangements and innovative institutional developments in sustainable food production and consumption. He is studying food consumption practices from a sociological perspective and is particularly interested in how consumers access sufficient, sustainable and healthy food.

Sigrid Wertheim-Heck is a professor of Food and Healthy Living at Aeres University of Applied Sciences and a senior research fellow at the Environmental Policy Group at Wageningen University, both in the Netherlands. Her interest in global urban food security informs her research on the relationship between metropolitan development, food provisioning and food consumption, focusing on equitable access to sustainable, safe and healthy foods. 